\title{
Técnica Transfisária Parcial para Plastia do Ligamento Cruzado Anterior em Idade Pediátrica
}

\section{Partial Transphyseal Technique for Anterior Cruciate Ligament Reconstruction in Skeletally Immature Patients}

Manuel Vieira-Silva, Rui M. Duarte ${ }^{1}$

Autor Correspondente:

\section{RESUMO}

Com o aumento da participação de crianças em desportos de competição em idades cada vez mais jovens, tem-se assistido a um aumento da incidência e do diagnóstico de roturas intrasubstância do ligamento cruzado anterior. À semelhança do adulto, a instabilidade ligamentar predispõe a criança a um potencial risco de lesões meniscais e condrais com consequentes alterações degenerativas precoces. O tratamento atempado destas lesões torna-se crucial, estando a técnica cirúrgica diretamente dependente da idade fisiológica do doente. Os autores apresentam um caso clínico de rotura completa do ligamento cruzado anterior em criança esqueleticamente imatura, tratada cirurgicamente por uma técnica transfisária parcial, com excelente resultado funcional.

PALAVRAS-CHAVE: Artroscopia; Criança; Desenvolvimento Ósseo; Ligamento Cruzado Anterior/cirurgia; Procedimentos Cirúrgicos Reconstrutivos

\section{ABSTRACT}

With more and more children participating in competitive sports at younger and younger ages, we have seen an increase in the incidence and diagnosis of intrasubstance ruptures of the anterior cruciate ligament.

As in adults, the instability of the ligaments predisposes children to a potential risk of meniscos and chondral injuries with consequent early degenerative alterations. The timely treatment of these injuries is crucial and the surgical technique depends directly on the patient's physiological age. The authors present a clinical case of the complete rupture of the anterior cruciate ligament in a skeletally immature child, surgically treated by a partial transphyseal technique, with an excellent functional result.

KEYWORDS: Anterior Cruciate Ligament/surgery; Arthroscopy; Bone Development; Child; Reconstructive Surgical Procedures 


\section{INTRODUÇÃO}

Nas últimas décadas tem-se verificado um aumento na prevalência da rotura do ligamento cruzado anterior (LCA) na população pediátrica com esqueleto imaturo.1,2 Tal deve-se principalmente a três fatores: i) o aumento da participação e um maior nível competitivo desportivo em crianças; ii) melhoria significativa das técnicas de imagem³; iii) maior alerta por parte da comunidade médica para esta temática. ${ }^{4}$

A atitude perante este tipo de lesões permanece controversa. A abordagem tradicional não cirúrgica defende um atraso no tratamento até que a criança atinja a maturidade esquelética, uma vez que a agressão da fise provocada pela construção dos túneis pode levar a perturbações do crescimento. ${ }^{5}$

No entanto, os dados reportados na literatura associam a instabilidade do joelho a um aumento progressivo e significativo de lesões intra-articulares, como por exemplo lesões condrais e meniscais. ${ }^{1,3}$

Em 2009 Henry J et al, ${ }^{6}$ apresentaram os resultados de um estudo comparativo de dois grupos de crianças (56 doentes) com rotura do LCA. O grupo 1 foi submetido a tratamento cirúrgico ad initium, enquanto que o grupo 2 foi inicialmente tratado conservadoramente até atingir a maturidade, e posteriormente submetido a plastia cirúrgica. $\bigcirc$ grupo 2 apresentava um número significativamente maior de lesões do menisco interno à data da cirurgia, assim como resultados significativamente piores ao nível funcional e clínico relativamente ao grupo 1.

Os dados da literatura demonstram que habitualmente a reconstrução cirúrgica do LCA (R-LCA) permite ao adolescente o regresso às suas atividades de vida diárias e desportivas habituais. Porém as técnicas habituais utilizadas no adulto cujos túneis (tibial e femoral) atravessam as fises com uma orientação oblíqua e excêntrica, ${ }^{1}$ são desaconselhadas. A perturbação da fise pode levar a distúrbios do crescimento, tais como deformidade angular do membro e/ou discrepâncias no comprimento., ${ }^{1,3}$

Este risco de lesão está diretamente relacionado com a maturidade da criança. Guzzanti V et al ${ }^{7}$ em 2003 estratificaram a população pediátrica com lesões do LCA em três grupos de acordo com a idade óssea, maturidade fisiológica e estadio da fise, de forma a prever o risco relativo de desenvolvimento de deformidades angulares e longitudinais após plastia do LCA.

De acordo com os seus trabalhos, os indivíduos com maior risco (pré-pubertários) apresentam uma idade óssea média de 12 anos, estadio I de Tanner, fises completamente abertas e um crescimento remanescente

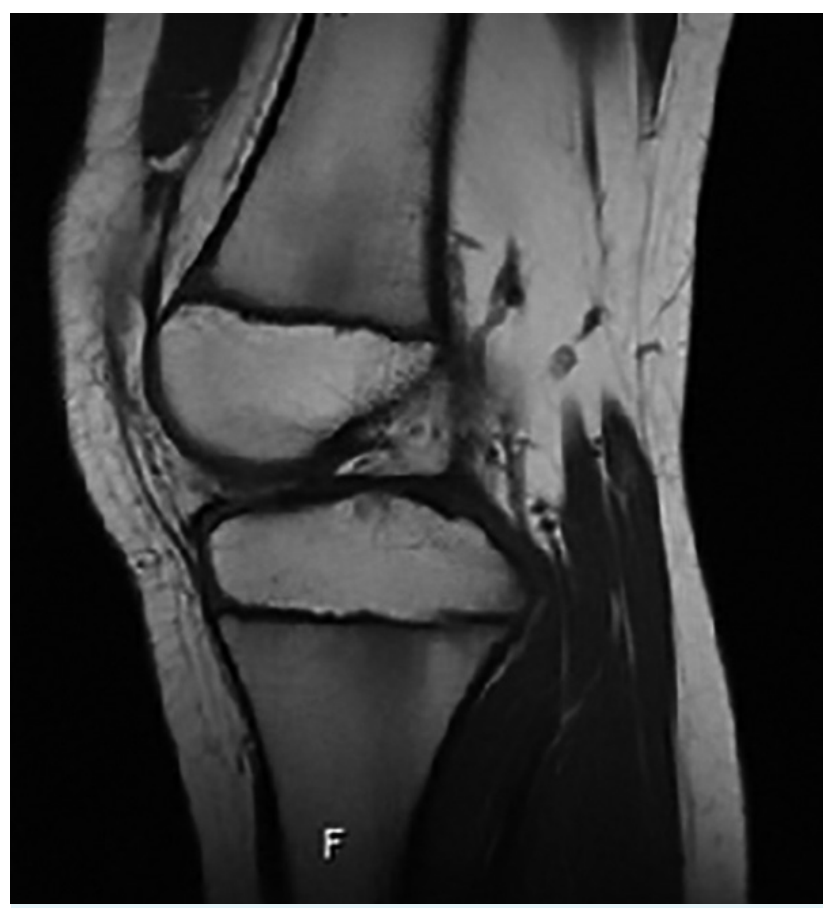

FIGURA 1. Imagem pré-operatória de RM (T2), evidenciando rotura intrasubstância do ligamento cruzado anterior.

potencial de $7 \mathrm{~cm}$. Apesar dos riscos associados ao procedimento cirúrgico, têm sido desenvolvidas técnicas de modo a minimizar o potencial risco de distúrbio do crescimento ao evitar a lesão das fises. ${ }^{8}$

Atualmente, as opções técnicas existentes para reconstrução do LCA incluem procedimentos poupadores da fise, ${ }^{2,7}$ procedimentos parcialmente transfisários ${ }^{8}$ e procedimentos completamente transfisários. ${ }^{3}$ Embora tecnicamente desafiadoras e ainda com poucos casos reportados na literatura, a reconstrução anatómica através de técnicas poupadoras da fise têm apresentado resultados promissores. Anderson AF,' reportou uma série de 12 doentes submetidos a reconstrução com túnel epifisário tibial e um túnel femoral efetuado sob controlo de fluoroscopia na face lateral da epífise femoral. As técnicas parcialmente transfisárias têm apresentado como principal vantagem uma melhor posição isométrica do enxerto tibial, evitando simultaneamente a perturbação da fise femoral. ${ }^{8}$ São descritos classicamente dois procedimentos: i) construção de túnel tibial transfisário e túnel femoral epifisário sob controlo de fluoroscopia ao nível do footprint do LCA (procedimento originalmente descrito por Lipscomb e Anderson em 198610); ii) construção de túnel tibial transfisário e fixação femoral numa posição over the top. ${ }^{8}$ Relativamente às técnicas completamente transfisárias apresentam a vantagem de serem muito semelhantes às reconstruções no adulto e desta forma familiares à maioria dos cirurgiões. Permitem um posicionamento isométrico dos túneis, podendo melhorar a longevidade do enxerto e a função do joelho. Recentemente têm sido 

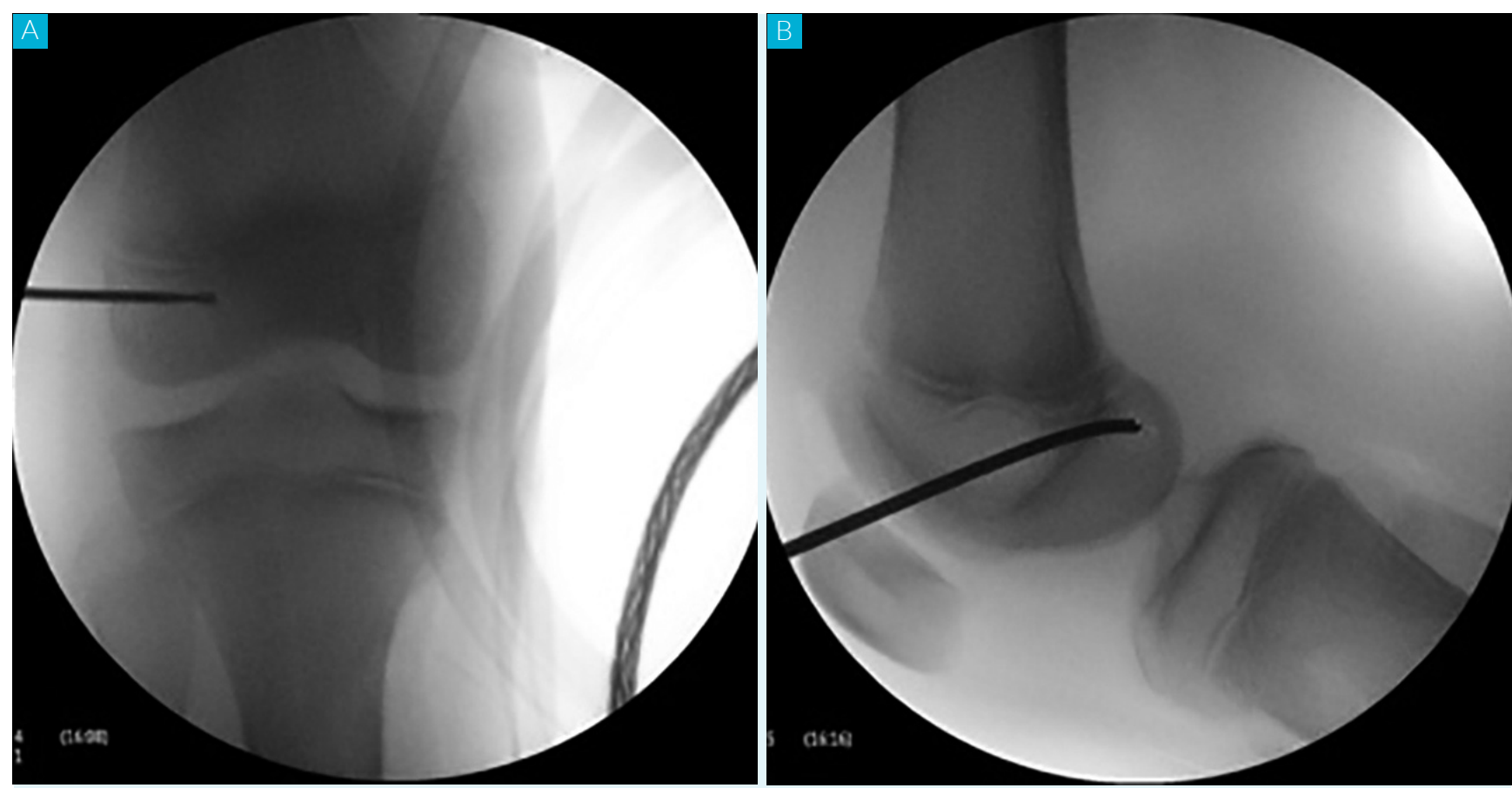

FIGURA 2. Imagem intra-operatória de fluoroscopia, observando-se:

A) Face - construção do túnel femoral abaixo da fise; B) Perfil - posicionamento posterior do túnel femoral.

publicados diversos resultados de reconstrução por técnica transfisária do LCA, destacando-se os trabalhos de Kocher MS,11 com 61 doentes operados nos estadios III e IV de Tanner, apresentando excelente resultado funcional (score IKDC e Lysholm de 89,5 e 91,2 respetivamente) e sem distúrbios do crescimento.

Independentemente da técnica utilizada, algumas recomendações devem ser tidas em conta: i) evitar tensionar excessivamente o enxerto; ii) os túneis transfisários devem ser completamente preenchidos por enxerto; iii) evitar fragmentos de osso ou hardware ao nível da fise; iv) os túneis transfisários devem ser pequenos (6-7 mm) e preferencialmente perpendiculares à fise, de forma a minimizar a agressão à placa de crescimento.

Os autores apresentam os resultados clínicos, funcionais e imagiológicos de um doente em estadio I de Tanner, submetidos a reconstrução do LCA por técnica parcialmente transfisária (epifisária femoral e transfisária tibial).

\section{CASO CLÍNICO}

Criança de 11 anos, sexo masculino com história de gonalgia direita após traumatismo durante a prática de futebol. O doente referia ter sofrido lesão rotacional, apresentando de seguida edema e incapacidade de marcha. Foi observado nos cuidados primários de saúde, tendo realizado radiografia do joelho. Foi medicado e aconselhado a realizar marcha apoiada com canadianas até melhoria do quadro clínico. Progressivamente ocorreu melhoria do edema e do componente álgico, no entanto foi incapaz de regressar à prática desportiva prévia por sensação de instabilidade. Por este motivo, três meses após o episódio traumático, foi referenciado para o nosso departamento de cirurgia do joelho.

Ao exame objetivo verificava-se discreto edema do joeIho direito, apresentando um arco de mobilidade normal mas doloroso na flexão máxima. Não foi verificada instabilidade lateral e teste de apreensão patelar foi negativo. Identificamos laxidez ântero-posterior na gaveta anterior, manobra de Lachman e pivot-shift, sugestivos de lesão do ligamento cruzado anterior (LCA).

A criança foi submetida a avaliação imagiológica com radiografia (filme extra-longo dos membros inferiores, face e perfil do joelho), revelando fises abertas, e ressonância magnética (RM) que demonstrou rotura completa do LCA (Fig. 1).

Após discussão do caso clínico com os pais, apresentação das hipóteses terapêuticas e esclarecimento dos riscos e benefícios, o doente e a família optaram pelo tratamento cirúrgico. À data da reconstrução do LCA, a criança encontrava-se no estadio I de Tanner e idade óssea de 11 anos. Com base na literatura, optou-se por uma técnica parcialmente transfisária com enxerto autólogo de tendões isquiotibiais.

Após a indução anestésica, o doente foi submetido a novo exame físico que confirmou insuficiência do LCA com manobras de Lachman e pivot-shift positivos. A artroscopia foi efetuada através dos portais standard ân- 
tero-medial e ântero-lateral, foi identificada uma rotura completa e intrasubstância do LCA, sem lesões meniscais ou condrais associadas. O remanescente do LCA foi removido e os footprints nativos identificados. Foram coIhidos os tendões semitendinoso e gracilis, pré-tencionados e preparados com sutura tipo Bunnel (Orthocord ${ }^{\circledR}$ \#2, Mitek). O túnel femoral (7 mm) foi construído por técnica outside-in sob controlo de fluoroscopia, de forma a evitar perturbação da fise (fio guia paralelo à fise) (Fig. 2). O túnel tibial (7 mm) por técnica transfisária foi construído também sob controlo de fluoroscopia, apresentando uma orientação perpendicular à fise. O enxerto foi introduzido através do fémur e a fixação femoral efetuada com parafuso de interferência absorvível 7×23 mm

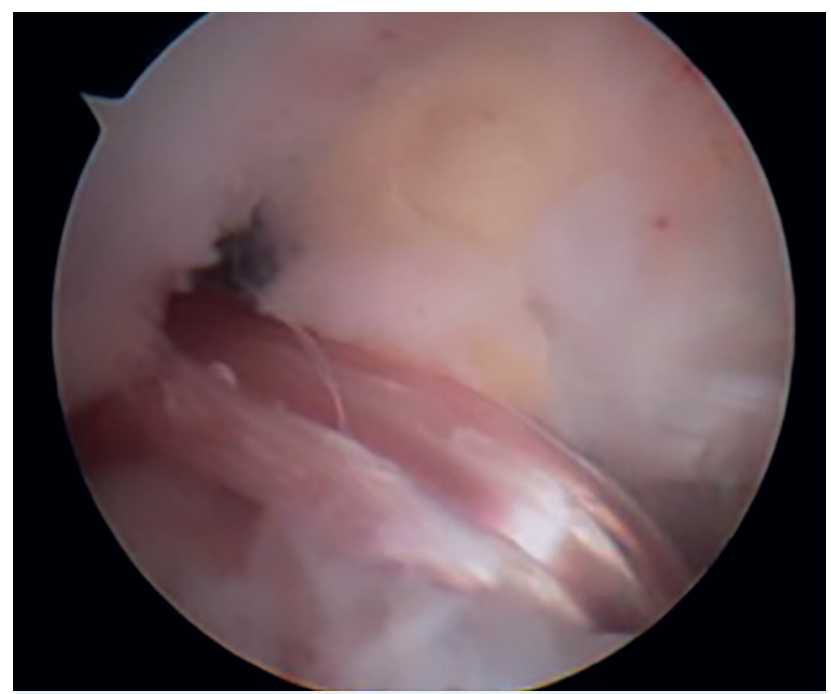

FIGURA 3. Imagem intra-operatória de artroscopia, observando-se o enxerto de isquiotibiais.

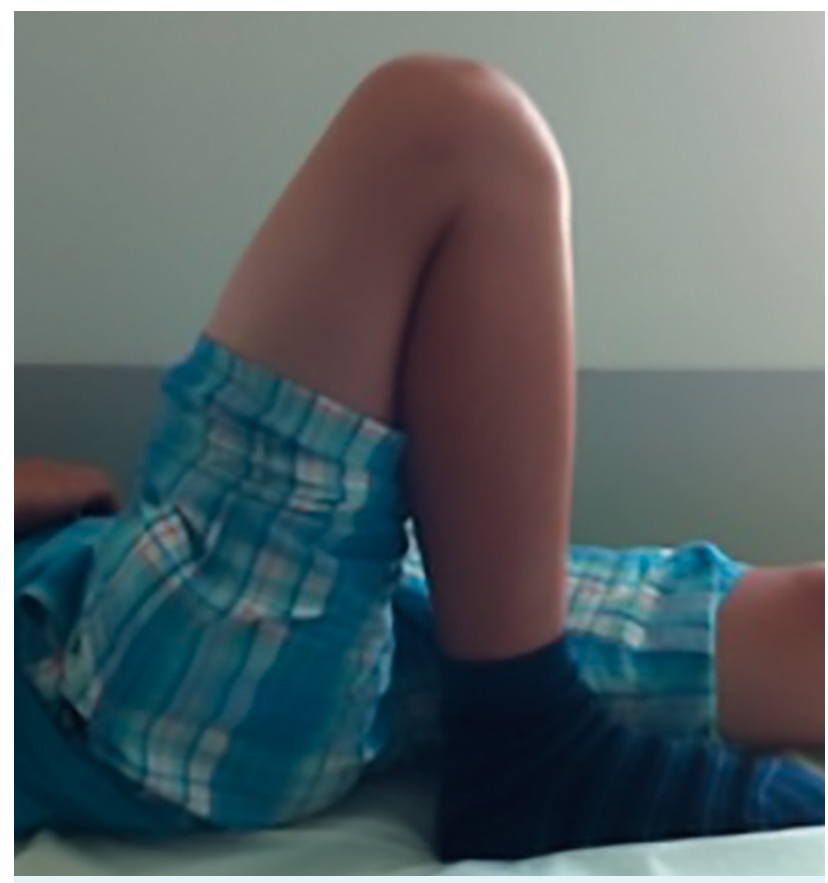

FIGURA 4. Fotografia de 1 ano pós-operatório, observando-se flexão completa de joelho.
(BioComposite ${ }^{\text {TM }}$, Arthrex) (Fig. 3). A fixação tibial foi efetuada com um grampo exterior ao túnel associado a parafuso tipo poste.

O pós-operatório decorreu sem intercorrências, tendo o doente iniciado o protocolo fisiátrico às duas semanas de pós-operatório, que manteve cerca de três meses. Regressou à atividade física prévia aos sete meses de pós-operatório. Aos 12 meses de pós-operatório encontra-se clinicamente bem, com arco de mobilidade completo (Fig. 4). Do ponto de vista funcional apresenta um score Lysholm de 95 e IKDC de 95,4. Mantém o acompanhamento em consulta externa com realização de estudo radiológico (alinhamento) (Fig. 5) de seis em seis meses até completar o crescimento.

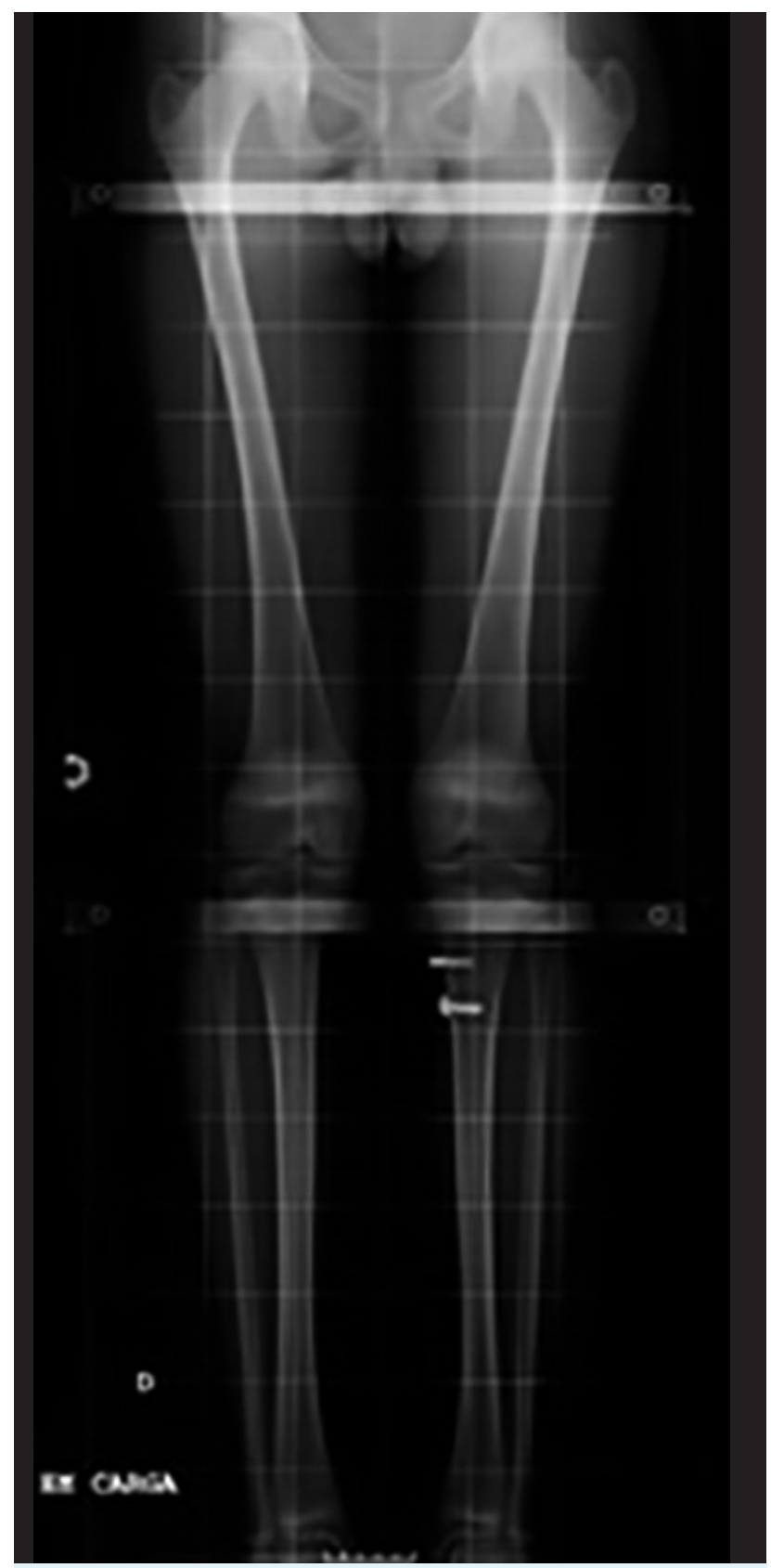

FIGURA 5. Imagem radiológica (extra-longo) de 1 ano pós-operatório, observando-se membros com alinhamento normal. 


\section{DISCUSSÃO}

Independentemente dos desenvolvimentos recentes no tratamento de roturas do LCA em doentes com imaturidade esquelética, várias questões continuam em aberto e permanecem controversas.

Embora a reconstrução do LCA seja consensual, de forma a manter a estabilidade e prevenir potenciais lesões futuras, o momento para o tratamento cirúrgico permanece em discussão. Deverá a reconstrução do LCA ser realizada durante a imaturidade esquelética ou após terminar o crescimento? Diversos estudos têm demonstrado um aumento das lesões secundárias, principalmente roturas do menisco interno em doentes submetidos a tratamento conservador até completarem o crescimento. ${ }^{6,12}$

Outra das questões em aberto será a opção pela técnica cirúrgica adequada. Apesar de serem reportados ótimos resultados com as três abordagens cirúrgicas - procedimentos poupadores da fise, procedimentos parcialmente transfisários e procedimentos completamente transfisários, ainda não é claro qual a abordagem adequada em função do estadio de desenvolvimento físico de Tanner. Na nossa opinião, as técnicas completamente transfisárias deverão ser reservadas para adolescentes já em fase final do crescimento (Tanner IV e V), pelo seu maior risco de perturbação do crescimento devido à lesão das fises. ${ }^{11,13}$ Quanto aos procedimentos poupadores da fise, habitualmente utilizados em crianças pré-pubertárias, englobam procedimentos combinados intra e extra-articulares que fornecem a vantagem de resultarem em risco reduzido de perturbação do crescimento. No entanto, alguns resultados menos favoráveis relativamente à laxidez clínica e instabilidade funcional associados aos procedimentos extra-articulares também têm sido reportados. $^{14}$

A opção por uma técnica híbrida (procedimento parcialmente transfisário) permanece uma excelente opção em estadios de Tanner I, II e III, permitindo que a fise femoral permaneça intacta, desta forma diminuindo o risco de compromisso do crescimento. Anderson $\mathrm{AF}^{9,10} \mathrm{e}$ Lipscomb $A B^{9,10}$ reportaram excelentes resultados a médio prazo (3 e 7 anos respetivamente) sem dismetrias ou deformidades observadas.

Em doentes com rotura completa do LCA e esqueleticamente imaturos, a avaliação do desenvolvimento fisiológico torna-se crucial para estratificar as diferentes opções cirúrgicas. A técnica parcialmente transfisária mostrou-se uma excelente opção no caso clínico apresentado.

CONFLITOS DE INTERESSE: Os autores declaram a inexistência de conflitos de interesse na realização do presente trabalho.
FONTES DE FINANCIAMENTO: Não existiram fontes externas de financiamento para a realização deste artigo.

PROTEÇÃO DE PESSOAS E ANIMAIS: Os autores declaram que os procedimentos seguidos estavam de acordo com os regulamentos estabelecidos pelos responsáveis. da Comissão de Investigação Clínica e Ética e de acordo com a Declaração de Helsínquia da Associação Médica Mundial.

CONFIDENCIALIDADE DOS DADOS: Os autores declaram ter seguido os protocolos do seu centro de trabaIho acerca da publicação dos dados de doentes.

\section{REFERÊNCIAS}

1. Greenberg EM, Albaugh J, Ganley TJ, Lawrence JT. Case report rehabilitation considerations for all epiphyseal $A C L$ reconstruction. Int J Sports Phys Ther. 2012;7:185-96.

2. Kocher MS, Garg S, Micheli LJ. Physeal sparing reconstruction of the anterior cruciate ligament in skeletally immature prepubescent children and adolescents. Surgical technique. J Bone Joint Surg. 2005;87:2371-9.

3. Redler LH, Brafman RT, Trentacosta N, Ahmad CS. Anterior cruciate ligament reconstruction in skeletally immature patients with transphyseal tunnels. Arthrosc. J Arthrosc Relat Surg. 2012;28:1710-7.

4. Accadbled F, Knörr J, Sales de Gauzy J. All inside transtibial arthroscopic posterior cruciate ligament reconstruction in skeletally immature: surgical technique and a case report. Orthop Traumatol Surg Res. 2013;99:361-5.

5. Frank JS, Gambacorta PL. Anterior cruciate ligament injuries in the skeletally immature athlete: diagnosis and management. J Am Acad Orthop Surg. 2013;21:78-87.

6. Henry J, Chotel F, Chouteau J, Fessy MH, Bérard J, Moyen B. Rupture of the anterior cruciate ligament in children: early reconstruction with open physes or delayed reconstruction to skeletal maturity? Knee Surgery Sport Traumatol Arthrosc. 2009;17:748-55.

7. Guzzanti V, Falciglia F, Stanitski CL. Physeal-sparing intraarticular anterior cruciate ligament reconstruction in preadolescents. Am J Sports Med. 2003;31:949-53.

8. McConkey MO, Bonasia DE, Amendola A. Pediatric anterior cruciate ligament reconstruction. Curr Rev Musculoskelet Med. 2011;4:37-44.

9. Anderson AF. Transepiphyseal replacement of the anterior cruciate ligament using quadruple hamstring grafts in skeletally immature patients. J Bone Joint Surg. 2004; 86 Suppl 1:201-9.

10. Lipscomb AB, Anderson AF. Tears of the anterior cruciate ligament in adolescents. J Bone Joint Surg. 1986;68:19-28.

11. Kocher M, Smith J, Zoric B, Lee B, Micheli L. Transphyseal anterior cruciate ligament reconstruction in skeletally immature pubescent adolescents. J Bone Joint Surg. 2007;89:2632-9.

12. Millett PJ, Willis AA, Warren RF. Associated injuries in pediatric and adolescent anterior cruciate ligament tears: does a delay in treatment increase the risk of meniscal tear? Arthroscopy. 2002;18:955-9.

13. Cohen M, Ferretti M, Quarteiro M, Marcondes FB, De Hollanda JP, Amaro JT, et al. Transphyseal anterior cruciate ligament reconstruction in patients with open physes. Arthroscopy. 2009;25:831-8.

14. Engebretsen L, Svenningsen S, Benum P. Poor results of anterior cruciate ligament repair in adolescence. Acta Orthop Scand. 1988;59:684-6. 